\title{
Yersinia enterocolitica and Chlamydia pneumoniae possible triggering agents of Guillain-Barré syndrome: a case report
}

\author{
Septimiu Tudor Bucurescu
}

Neurology at Vital-Klinik, Bad Driburg, Germany

Article Info

\section{Article Notes}

Received: August 31, 2017

Accepted: March 17, 2018

${ }^{*}$ Correspondence:

Dr. Septimiu Bucurescu

Neurology at Vital-Klinik, Bahnhofstr. 3, 33014 Bad Driburg, Germany, Email: s_bucurescu@yahoo.com

C) 2018 Bucurescu ST. This article is distributed under the terms of the Creative Commons Attribution 4.0 International License

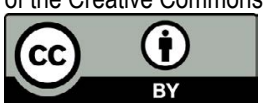

\section{Keywords:}

Guillain-Barré syndrome triggering agent

Yersinia enterocolitica

Chlamydia pneumoniae

anti-ganglioside antibody induction

\section{ABSTRACT}

In this paper we describe the case of a Guillain-Barré syndrome patient who was diagnosed with an active yersiniosis and past chlamydiosis. We also discuss the diagnosis, therapy and recovery prognosis of patients with Guillain-Barré syndrome.

\section{Introduction}

Guillain-Barré syndrome is an self-limited, acute inflammatory demyelinating polyradiculoneuropathy usually preceded by a bacterial or viral infection. The disease has usually three evolutionary phases: in the first phase, lasting two to four weeks, symptoms worsen, in the second phase, lasting up to several weeks, symptoms stabilize and in the third phase, lasting up to several months symptoms improve ${ }^{1}$. Overall incidence of the disease is low: 0.34-1.34/100,000/year in children under 15 years of age, 1.1-1.8/100,000/year in adults under 50 years of age and 1.7-3.3/100,000/year in adults over 50 years of age ${ }^{2}$. The economic burden of disease in the United States only was estimated at $\$ 1.7$ billion yearly ${ }^{3}$. Although the most frequently identified triggering agent of Guillain-Barré syndrome is Campylobacter jejuni, the disease is considered to be an idiopathic disease ${ }^{4}$. Diagnosis is based on clinical history, neurological and laboratory examination ${ }^{5}$. Treatment recommendations are plasma exchange for 6 to 10 days $^{6}$ or intravenous immunoglobulin for 5 days $^{7}$. Recovery can be calculated using a clinical prognostic scoring system ${ }^{8}$; mortality is between $3-10 \%$ and disability after 6 months $20 \%{ }^{9}$. The average length of hospital stay is 34 days in acute care and 26 days in rehabilitation ${ }^{10}$.

\section{Patient history}

A 38-year-old man was admitted to the emergency department of an acute care hospital with severe tetraparesis and hypoesthesia. The symptoms occurred 2 days before hospital admission, 10 days after influenza infection. The anamnesis provided information about previous diseases, sinusitis 2 months ago with nasal congestion and pain, and gastroenteritis 10 months ago with abdominal pain and diarrhea, as well as vaccination status, the patient was not vaccinated against influenza. The patient was not taking any fixed-schedule medication, only ibuprofen on-demand. The clinical examination (i.e., general, neurological and psychopathological) showed tetraparesis, hypoesthesia and absent tendon reflexes. A standard laboratory blood test was done. All investigated parameters (i.e., sodium, potassium, urea, gamma-glutamyl transferase, hemoglobin, hematocrit, international 
normalized ratio, creatinine, mean corpuscular hemoglobin, mean corpuscular hemoglobin concentration, mean corpuscular volume, activated partial thromboplastin time, thrombocytes, erythrocytes, leucocytes, creatine kinase, calcium, lactate dehydrogenase, glutamic oxaloacetic transaminase, hemoglobin A1c and thyroid stimulating hormone) were normal. The cerebrospinal fluid analysis (i.e., proteins, glucose, red blood cells, white blood cells, Reiber nomograms) showed normal values. ECG showed no pathological findings. Electroneurographic examination of upper and lower limbs (i.e., median, ulnar, tibial and peroneal nerve) showed normal F-wave, normal distal motor latency, and normal motor and sensory velocity of nerve conduction. Another electroneurographic examination done 2 days after hospital admission showed absence of F-wave, motor but not sensory conduction block, findings that are typical for an acute demyelinating neuropathy. The patient was diagnosed with Guillain-Barré syndrome and received intravenous immunoglobulin for 5 days. Symptoms improved after treatment.

After acute care the patient was transferred to our clinic for neurological rehabilitation. The clinical examination showed tetraparesis and hypoesthesia, tendon reflexes were present. Barthel activities of daily living index was 70 points $(0-100)$. A standard laboratory blood test was done. The values of glutamic oxaloacetic transaminase $69 \mathrm{U} / \mathrm{L}(<50)$, alanine aminotransferase $114 \mathrm{U} / \mathrm{L}(<50)$, potassium $4.9 \mathrm{mmol} / \mathrm{L}$ (3.5-4.5), cholesterol $210 \mathrm{mg} / \mathrm{dL}$ $(<200)$ were increased. The value of HDL-cholesterol 36 $\mathrm{mg} / \mathrm{dL}(>40)$ was decreased. In addition to the standard laboratory blood test, microbiological blood and stool tests were done. Blood ELISA-tests were negative for Yersinia enterocolitica IgG 0,681 ratio $(<0.8)$ and positive for $\operatorname{IgA}$ 1.181 ratio $(<0.8)$ antibodies, results suggesting an active infection. Blood ELISA-tests were positive for Chlamydia pneumoniae IgG 2.145 ratio $(<0.8)$ and negative for IgA 0.6 ratio $(<0.8)$ antibodies, results suggesting a previous infection. Blood ELISA-tests were positive for Mycoplasma pneumoniae IgG 1.538 ratio $(<0.8)$ and borderline for IgA 0.863 ratio $(0.8-1.1)$, results suggesting a questionable acute infection. Stool antigen detection of Campylobacter, and stool culture of Salmonella, Shigella, Yersinia and Campylobacter were negative. The patient received physiotherapy for 4 weeks which improved his condition. During neurological rehabilitation no drug therapy was necessary. Barthel activities of daily living index was 95 points (0-100). Since the patient was not completely recovered, he was supposed to continue ambulatory physiotherapy after discharge.

\section{Discussions}

Campylobacter jejuni is a leading cause of acute gastroenteritis $^{11}$. Campylobacter jejuni is triggering Guillain-Barré syndrome by anti-ganglioside antibody induction due to molecular mimicry between bacterial cell wall lipooligosaccharides and myelin sheath oligosaccharide core of gangliosides, which are neuronal membrane glycolipids ${ }^{12}$.Yersinia enterocolitica causes most often acute and chronic gastroenteritis ${ }^{13}$. In the bacterial cell wall of human strains lipopolysaccharides have been detected and characterized ${ }^{14}$. Chlamydia pneumoniae is a common cause of upper and lower respiratory tract infections ${ }^{15}$. In the bacterial cell wall lipopolysaccharides have been detected and characterized ${ }^{16}$. A case report has been published suggesting that Mycoplasma pneumoniae could trigger Guillain-Barré syndrome ${ }^{17}$. There are no published case reports on yersiniosis and/or chlamydiosis preceding Guillain-Barré syndrome. There is no evidence in the biomedical literature that Yersinia enterocolitica and/or Chlamydia pneumoniae bacterial cell wall lipopolysaccharides are inducing anti-ganglioside antibody response.

\section{Conclusions and future perspectives}

In order to elucidate whether Yersinia enterocolitica and/or Chlamydia pneumoniae are triggering agents of Guillain-Barré syndrome or not, future studies on mice must to be done. Anti-ganglioside antibody induction by Campylobacter jejuni lipooligosaccharides was proven by immunization studies in mice ${ }^{18}$. Mice were immunized with Campylobacter jejuni lipopolysaccharides isolated from patients with Miller-Fisher syndrome, a variant of Guillain-Barré syndrome, and anti-ganglioside mice antibodies were cloned. Mice antibodies were capable of binding to the nerve terminal and cause complementmediated paralysis in ex-vivo muscle nerve preparation. This approach could be used to investigate whether Yersinia enterocolitica and/or Chlamydia pneumoniae bacterial cell wall lipopolysaccharides are inducing antiganglioside antibody response or not.

Clinical studies must be done in order to clarify whether adult patients with Guillain-Barré syndrome and positive blood tests for above mentioned bacteria could benefit from antibiotic treatment in addition to plasma exchange or intravenous immunoglobulin. There are no published studies on antibiotics use in GuillainBarré syndrome. Patients with positive blood tests for Campylobacter jejuni could receive erythromycin $500 \mathrm{mg}$ twice daily for 5 days or azithromycin $500 \mathrm{mg}$ daily for 3 days $^{19}$. Patients with positive blood tests for Yersinia enterocolitica could receive trimethoprim/ sulfamethoxazole $160 / 800 \mathrm{mg}$ twice daily for at least 7-10 days $^{20}$. Patients with positive blood tests for Chlamydia pneumoniae could receive tetracycline $500 \mathrm{mg}$ four times daily for 14 days, doxycycline $100 \mathrm{mg}$ twice daily for 14 days or erythromycin $500 \mathrm{mg}$ four times daily for 14 days $^{21}$. Reduced mortality and/or length of hospital stay should reduce also the economic burden of disease. 


\section{References}

1. Hahn AF. Guillain-Barré syndrome. Lancet. 1998; 352(9128): 635641.

2. McGrogan A, Madle GC, Seaman HE, et al. The epidemiology of Guillain-Barré syndrome worldwide. A systematic literature review. Neuroepidemiology. 2009; 32(2): 150-163.

3. Frenzen PD. Economic cost of Guillain-Barré syndrome in the United States. Neurology. 2008; 71: 21-27.

4. Godschalk PC, Heikema AP, Gilbert M, et al. The crucial role of Campylobacter jejuni genes in anti-ganglioside antibody induction in Guillain-Barré syndrome. J Clin Invest. 2004; 114(11): 1659-1665.

5. Fokke C, van den Berg B, Drenthen J, et al. Diagnosis of Guillain-Barré syndrome and validation of Brighton criteria. Brain. 2014; 137(Pt 1) 33-43

6. Raphael J, Chevret S, Hughes R, et al. Plasma exchange for GuillainBarré syndrome. Cochrane Database Syst Rev. 7, CD001798.

7. Hughes R, Swan A, van Dorn P. Intravenous immunoglobulin for Guillain-Barré syndrome. Cochrane Database Syst Rev. 9, CD002063.

8. van Koningsveld R, Steyerberg EW, Hughes RA, et al. A clinical prognostic scoring system for Guillain-Barré syndrome. Lancet Neurol. 2007; 6: 589-594.

9. van Doorn PA, Ruts L, Jacobs BC. Clinical features, pathogenesis, and treatment of Guillain-Barré syndrome. Lancet Neurol. 2008; 7(10): 939-950.

10. Meythaler JM, DeVivo MJ, Braswell WC. Rehabilitation outcomes of patients who have developed Guillain-Barré syndrome. Am J Phys Med Rehabil. 1997; 76(5): 411-419.

11. Fitzgerald C. Campylobacter. Clin Lab Med. 2015; 35(2): 289-298.
12. Ang CW, Jacobs BC, Laman JD. The Guillain-Barré syndrome: a true case of molecular mimicry. Trends Immunol. 2004; 25: 61-66.

13. Saebo A, Lassen J. Acute and chronic gastrointestinal manifestations associated with Yersinia enterocolitica infection. A Norwegian 10year follow-up study on 458 hospitalized patients. Ann Surg. 1992; 215(3): 250-255.

14. Bialas N, Kasperkiewicz K, Radziejewska-Lebrecht J, et al. Bacterial cell surface structures in Yersinia enterocolitica. Arch Immunol Ther Exp. 2012; 60: 199-209.

15. Grayston JT, Campbell LA, Kuo CC, et al. A new respiratory tract pathogen: Chlamydia pneumonia strain TWAR. J Infect Dis. 1990; 161(14): 618-625.

16. Brade H, Brade L, Nano FE. Chemical and serological investigations on the genus-specific lipopolysaccharide epitope of Chlamydia. Proc Natl Acad Sci USA. 1987; 84(8): 2508-2512.

17. Meyer Sauteur PM, Huizinga R, Tio-Gillen AP, et al. Mycoplasma pneumoniae triggering the Guillain-Barré syndrome: a case-control study. Ann Neurol. 2016; 80(4): 566-580.

18. Goodyear CS, O’Hanlon GH, Plomp JJ, et al. Monoclonal antibodies raised against Guillain-Barré syndrome-assiciated Campylobacter jejunii lipopolysaccharides react with neuronal gangliosides and paralyze muscle-nerve preparations. J Clin Invest. 1999; 104(6): 697 708.

19. Allos BM. Campylobacter jejuni infections: update on emerging issues and trends. Clin Infect Dis. 2001; 32(8): 1201-1206.

20. Hoogkamp-Korstanje JA. Antibiotics in Yersinia enterocolitica infections. J Antimicrob Chemother. 1987; 20(1): 123-131.

21. Blasi F, Tarsia P, Aliberti S. Chlamydophilia pneumoniae. Clin Microbiol Infect. 2009; 15(1): 29-35. 